\title{
Spherical Neutralizing Aptamer Inhibits SARS-CoV-2 Infection
}

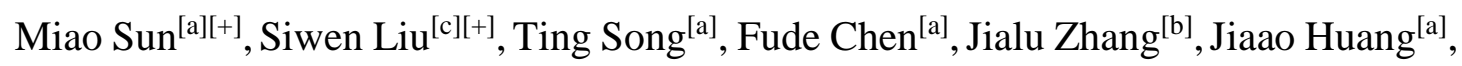
Shuang Wan ${ }^{[\mathrm{a}]}$, Yao Lu ${ }^{[\mathrm{a}]}$, Honglin Chen ${ }^{[\mathrm{c}] *}$, Weihong $\operatorname{Tan}^{[\mathrm{b}]}$, Yanling Song ${ }^{[\mathrm{a}] *}$, and Chaoyong Yang ${ }^{[\mathrm{a}][\mathrm{b}] *}$

\begin{abstract}
New neutralizing agents against SARS-CoV-2 and the associated mutant strains are urgently needed for the treatment and prophylaxis of COVID19. Herein, we develop a spherical cocktail neutralizing aptamer-gold nanoparticle (SNAP) to synergistically block the interaction of SARS$\mathrm{CoV}-2$ receptor-binding domain (RBD) and angiotensin-converting enzyme-2 (ACE2). Taking advantage of the simultaneous recognition of multi-homologous and multi-heterogenous neutralizing aptamers and dimensionally matched nano-scaffolds, the SNAP exhibits increased affinity to the RBD with a dissociation constant value of $5.46 \mathrm{pM}$ and potent neutralization against authentic SARS-CoV-2 with a half-maximal inhibitory concentration of $142.80 \mathrm{aM}$. Additional benefits include the multi-epitope blocking capability of the aptamer cocktail and the steric hindrance of the nano-scaffold, which further covers the ACE2 binding interfaces and affects the conformational transition of the spike protein. As a result, the SNAP strategy exhibits broad neutralizing activity, almost completely blocking the infection of N501Y and D614G mutant strains. Overall, the SNAP strategy provides a new direction for development of anti-virus infection mechanisms, both to fight the COVID-19 pandemic and serve as a powerful technical reserve for future unknown pandemics.
\end{abstract} [a] The MOE Key Laboratory of Spectrochemical Analysis \& Instrumentation, the Key Laboratory of
Chemical Biology of Fujian Province, State Key Laboratory of Physical Chemistry of Solid Surfaces,
Department of Chemical Biology, College of Chemistry and Chemical Engineering, Xiamen University,
Xiamen, Fujian 361005, China. [b] Institute of Molecular Medicine, Renji Hospital, Shanghai Jiao
Tong University School of Medicine, Shanghai Jiao Tong University, Shanghai, 200127, China. [c]
State Key Laboratory for Emerging Infectious Diseases and InnoHK Centre for Infectious Diseases,
Department of Microbiology, Li Ka Shing Faculty of Medicine, the University of Hong Kong, Hong
Kong SAR, China.
These authors contributed equally to this work: Miao Sun, Siwen Liu
Corresponding author E-mail: hlchen@hku.hk; ylsong@xmu.edu.cn; cyyang@xmu.edu.cn 


\section{Main}

The global pandemic of SARS-CoV-2 and the associated COVID-19 disease has emphasized the importance of developing new therapeutics against novel viruses. The entrance of SARS-CoV-2 into host cells is mediated by the RBD of trimeric-spike (S-RBD) binding to ACE2 expressed on the host cells. ${ }^{1,2}$ At present, some neutralizing antibodies against SARS-CoV-2 are being developed to block viral entry by binding to the ACE2 interaction interfaces on the S-RBD. ${ }^{3}$ However, as an RNA virus, SARS-CoV-2 has a high mutation rate, leading to the emergence of many mutant strains such as D614G and N501Y, ${ }^{4,5}$ which may escape the therapy of the existing neutralizing antibodies. ${ }^{6,7}$ Moreover, most neutralizing antibodies against SARS-CoV-2 are derived from animal immunizations and convalescent serum, both of which involve complex and time-consuming procedures, and may not result in sufficient immunity. ${ }^{8,9}$ Therefore, alternative novel strategies to rapidly produce potent neutralizing biologics that are broadly effective against SARS-CoV2 and mutants, as well as future related coronaviruses, are urgently needed.

In vitro selection for neutralizing aptamers against SARS-CoV-2 can be performed without infected individuals, and only proteins are needed as targets. ${ }^{10,11}$ Compared to neutralizing antibodies $(\sim 150 \mathrm{kDa})$, the smaller size of neutralizing aptamers $(\sim 15 \mathrm{kDa})$ allows them to penetrate tissues that may not be accessible to classical antibodies. In addition, neutralizing aptamers can be easily and rapidly produced by chemical synthesis without batch-to-batch differences. ${ }^{12-14}$ However, as monomers, neutralizing aptamers cannot ensure simultaneous binding of the three $\mathrm{RBD}_{\mathrm{S}}$ on the trimeric-spike, resulting in unsatisfactory therapeutic application.

To form a simultaneous interaction, we engineered spherical cocktail neutralizing aptamer-gold nanoparticle (SNAP) conjugates to synergistically block the trimeric-spike thus inhibiting SARS-CoV-2 infection. SNAP strategy offers several unique advantages. First, homoand hetero-neutralizing aptamers can be easily anchored with high density and homogeneous orientation on the same gold-nanoparticle ${ }^{15,16}$, affording improved binding affinity and simultaneous blocking of different RBDs on the trimeric-spike. Second, the particle scaffold can provide a 
dimensionally matched interaction between spherical neutralizing aptamers $(\sim 5 \mathrm{~nm})$ and the trimeric-spike $(\sim 5 \mathrm{~nm})$. The resultant spatially organized interaction effectively adds a "hat" to the trimeric-spike's apex of S-RBD. The nanoscale "hat" not only largely covers the ACE2 binding interfaces on the S-RBD, but also affects the conformational transition of the trimeric-spike, which is another crucial step associated with infection and pathogenicity ${ }^{17}$, as well as the interaction of S-RBD and ACE2. Therefore, SNAP provides appropriate steric hindrance against both viral recognition and fusogenic conformational formation, resulting in excellent neutralization potency. Finally, gold nanoparticles provide aptamers with a reduced the propensity toward nuclease degradation compared to the linear aptamer counterparts. ${ }^{15,18}$ Therefore, multi-homologous and multiheterogenous aptamers on the SNAP conjugate are expected to target not only distinct epitopes of RBD monomers within a trimeric-spike, but also different $\mathrm{RBD}_{\mathrm{S}}$ on the trimeric-spike, thus inhibiting the infection of SARS-CoV-2 with ideal potency.

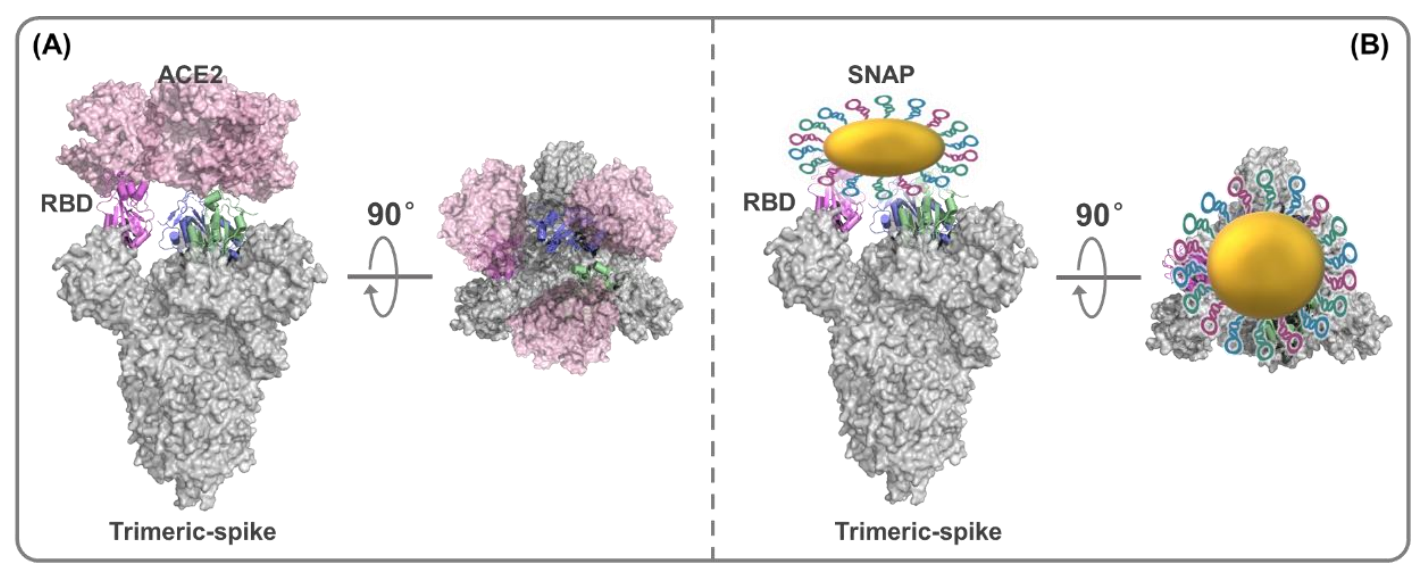

Scheme 1. The mechanism of (A) SARS-CoV-2 infection by interaction of trimericspike and ACE2. The three RBD in the trimeric-spike (grey) are depicted in violet, green, and pink, respectively. ACE2 is depicted in light pink. (B) The mechanism of SARS-CoV-2 neutralization by SNAP. SNAP is depicted in gold, and the three neutralizing aptamers depicted in blue, green, and pink, respectively.

Since SARS-CoV-2 is known to mutate, to avoid the increased risk of mutation-induced neutralization escape, it is crucial to apply a potent series of neutralizing aptamers as a cocktail for therapy. To form multiheterogenous binding, three aptamers (CoV2-1C, CoV2-4C, and CoV26C3) ${ }^{10,11}$, each targeting SARS-CoV-2, were chosen for SNAP formation. The results of cross-competition experiments (Figure 1A) and the reported 
molecular dynamics simulations ${ }^{10,11}$ (Figure 1B) suggest that these three aptamers have very little interference with each other in RBD recognition and interact with three non-overlapping epitopes on RBD.

We then investigated the neutralization efficiency by determining the binding tendency of ACE2 with RBD with or without pre-blocking by the individual aptamers or the aptamer cocktail. Consistent with the predicted results, the aptamer cocktail has the highest RBD neutralization efficiency compared to the any of the single aptamers (Figure 1C). The RBD neutralization efficiency of aptamer cocktail is 10.3 times, 2.7 times, and 1.4 times that of $\mathrm{CoV} 2-1 \mathrm{C}, \mathrm{CoV} 2-4 \mathrm{C}$, and $\mathrm{CoV} 2-6 \mathrm{C} 3$ aptamers, respectively. It is worth noting that although $\mathrm{CoV} 2-6 \mathrm{C} 3$ aptamer plays the leading role of inhibition, CoV2-1C (dissociation constant $\left(\mathrm{K}_{\mathrm{d}}\right)$ value of $5.8 \mathrm{nM}){ }^{10}$ and CoV2-4C ( $\mathrm{K}_{\mathrm{d}}$ of $\left.19.9 \mathrm{nM}\right){ }^{10}$ aptamers have better RBD binding affinities than that of CoV2-6C 3 aptamer $\left(\mathrm{K}_{\mathrm{d}}\right.$ of $\left.44.8 \mathrm{nM}\right){ }^{11}$. Thus, addition of CoV2-1C and CoV2-4C aptamers not only reduces the escape of mutant strains, but also improves the recognition dynamics of SNAP.
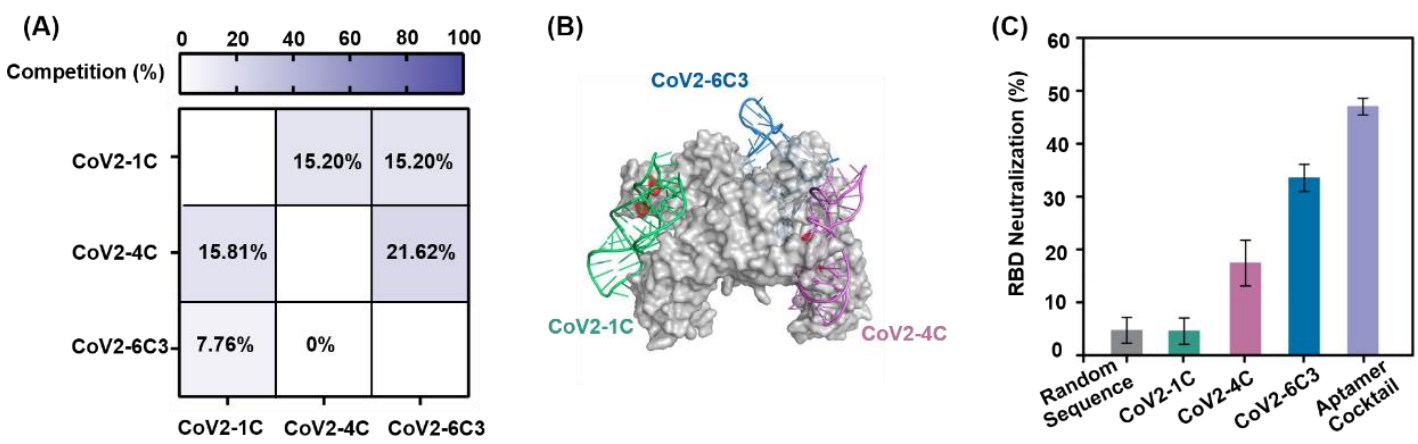

Figure 1. (A) The results of cross-competition experiments between any two of CoV2$1 \mathrm{C}, \mathrm{CoV} 2-4 \mathrm{C}$ and $\mathrm{CoV} 2-6 \mathrm{C} 3$ aptamers. One fluoresces labeled aptamers were mixed two unlabeled aptamers and RBD-beads were incubated, washed and analyzed by flow cytometry. The percentage of the cross-competition was calculated by the equation (the fluorescence signal of the fluorescent aptamer with the other two non-labeled aptamers - background signal) / the fluorescence signal of the fluorescent aptamer without the other two non-labeled aptamers - background signal). (B) Molecular dynamics simulated binding modes of $\mathrm{CoV} 2-1 \mathrm{C}, \mathrm{CoV} 2-4 \mathrm{C}$ and $\mathrm{CoV} 2-6 \mathrm{C} 3$ aptamers against SARS-CoV-2 RBD (in grey). The ACE2 binding amino acids on RBD are red. (C) RBD neutralization efficiency of candidate sequences and aptamer cocktail. The percentage of RBD neutralization in the presence of $\mathrm{CoV} 2-1 \mathrm{C}, \mathrm{CoV} 2-4 \mathrm{C}$ and $\mathrm{CoV} 2-6 \mathrm{C} 3$ was compared to RBD binding without aptamers, calculating by the equation (1- (the fluorescence signal of the sample with the aptamer incubation - background signal) / (the fluorescence signal of the sample without the aptamer incubation - background 
signal) $* 100 \%)$

To improve binding affinity and neutralization potency, we utilized $5 \mathrm{~nm}$ gold nanoparticles (AuNPs) as the scaffold to assemble the aptamer cocktail and form SNAP conjugates, with the goal of blocking multiple RBDs on the same spike simultaneously. AuNPs were functionalized with SH-labeled cocktail aptamers by the freeze-thaw method. ${ }^{19}$ As shown in the results of transmission electron microscopy (Fig 2A and B) and dynamic light scattering (Fig 2C and D), the SNAP conjugate has a slightly larger diameter and a smaller polydispersity index value than those of bare AuNPs, suggesting the successful modification and good monodispersibility of SNAP conjugates. The aptamer number on each AuNP was evaluated to be ca. 340, guaranteeing multivalent binding for better neutralization. The $\mathrm{K}_{\mathrm{d}}$ value of SNAP conjugate against RBD protein was found to be $5.46 \mathrm{pM}$, about 1000-8000-fold higher affinity than the standalone aptamers ${ }^{10,11}$. Moreover, SNAP also recognizes the SARS-CoV-2 spike protein (Figure S2), but no to the RBD or spike protein of other viruses (Figure S3), indicating the ideal universality and selectivity of SNAP. In addition to multivalent interaction, the dense oligonucleotide shell of the SNAP conjugate can deactivate the enzymes in close proximity to the aptamers due to the high local sodium ion concentration. Therefore, SNAP can evade nucleases, resulting in a decreased rate of hydrolysis compared to the single aptamers (Figure $2 \mathrm{G}$ and $\mathrm{H}$ ), thereby improving the stability in vivo.

(A)

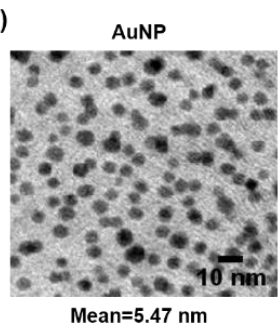

(E)

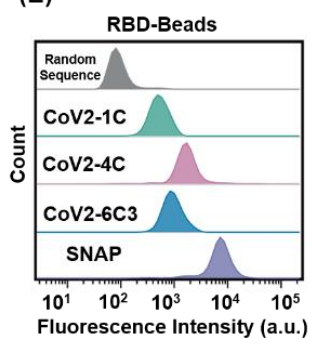

(B)

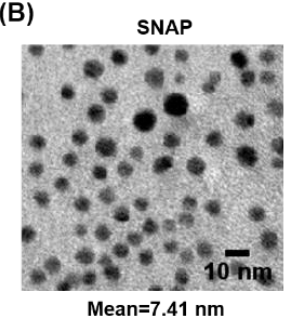

(F)

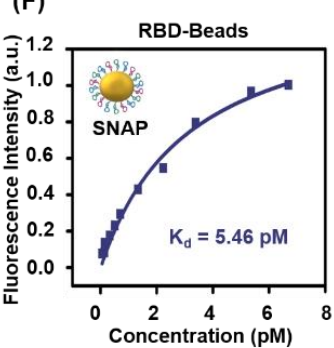

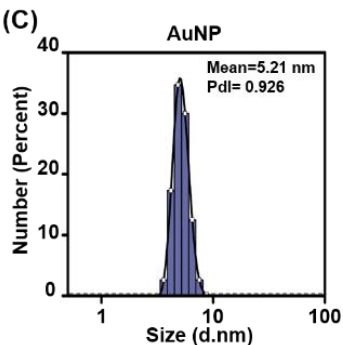

(G)

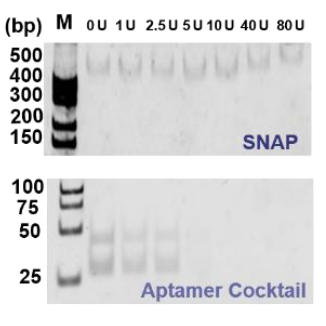

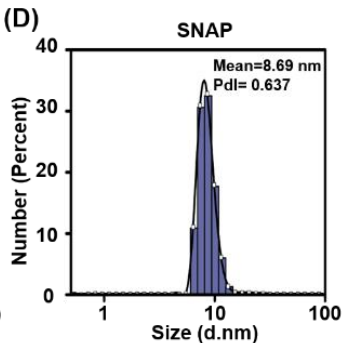

(H)

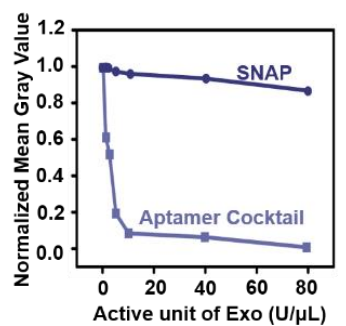

Figure 2. Transmission electron microscopy images of the AuNPs (A) and SNAPs (B). 
Size distribution histograms of the AuNPs (C) and SNAPs (D) analyzed by dynamic light scattering. (E) Flow cytometry analysis of monomer aptamers and SNAP against RBD-beads. (F) Binding curve of SNAP against RBD protein. (G) Stability analysis of SNAP (upper) and aptamer cocktail (lower) after incubation with different concentrations of Exo I, as determined by agarose electrophoresis. $(\mathrm{H})$ Analysis of agarose gel characterization of the stability of SNAP and aptamer cocktail based on the normalized mean gray value (calculated by ImageJ).

To confirm the neutralization of virus infection by SNAP inhibition of S-RBD and ACE2 interaction, we utilized a pseudotyped virus, which was packed with the SARS-CoV-2 trimetric-spike and contained the plasmid consisting of a GFP and a luciferase reporter. As shown in Fig 3A, SNAPs protected more than $96 \%$ of the cells from pseudotyped SARSCoV-2 infection, displaying much higher potency than the respective aptamer cocktail (64.52\%). This success is attributed to the simultaneous interaction of SNAP with multiple epitopes on multiple RBDs within the trimeric-spike using homo- and hetero-aptamers, thus more fully occluding the ACE2 binding interfaces via the AuNP scaffold. In addition, the neutralizing efficiency of SNAP is 1.3 times that of CoV2-6C3-AuNP, indicating that the $\mathrm{CoV} 2-1 \mathrm{C}$ and $\mathrm{CoV} 2-4 \mathrm{C}$ aptamers are also useful in the overall binding to RBD and inhibition of the virus infection, possibly due to the higher binding affinities of $\mathrm{CoV} 2-1 \mathrm{C}$ and $\mathrm{CoV} 2-4 \mathrm{C}$ aptamers. It is also worth noting that even for the CoV2-1C aptamer with a weak inhibition ability (39.22\%), the inhibition effect was obviously improved after incorporation of CoV2-1C-AuNP with the $5 \mathrm{~nm}$ gold-scaffold $(50.83 \%)$. The inhibition ability of SNAP $(96.16 \%)$ is also greater than that of a commercial neutralizing antibody (77.52\%, Research Resource Identifiers Number: AB_2857935) at the same concentration. And SNAP conjugates exhibited excellent inhibitory activity against pseudotyped SARS-CoV-2 virus with a half-maximal inhibitory concentration $\left(\mathrm{IC}_{50}\right)$ of $207.70 \mathrm{fM}$ (Figure 3C), which is about four orders of magnitude smaller than CoV2-6C3 aptamer. Moreover, as reported in the literature of $5 \mathrm{~nm}$ gold nanoparticles ${ }^{20,21}$, SNAP showed no obvious cytotoxicity (Figure S4) and immunogenicity (Figure S5 and S6). 
Cryo-electron microscopy imaging was carried out to validate the interaction between SNAP and the pseudovirus. SNAP acts as a nanoscale "hat" to cover the spike of the pseudovirus, resulting in a slight deformation of the spike protein (Figure 3B, Figure S7). Overall, based on neutralization by the aptamer cocktail and the steric effect of the gold scaffold, which hinders the interaction of trimeric-spike-ACE2 and the conformational transition of the trimeric-spike, the SNAP strategy is highly likely to enable synergistic-blocking of the trimeric-spike for potent inhibition of SARS-CoV-2 infection.

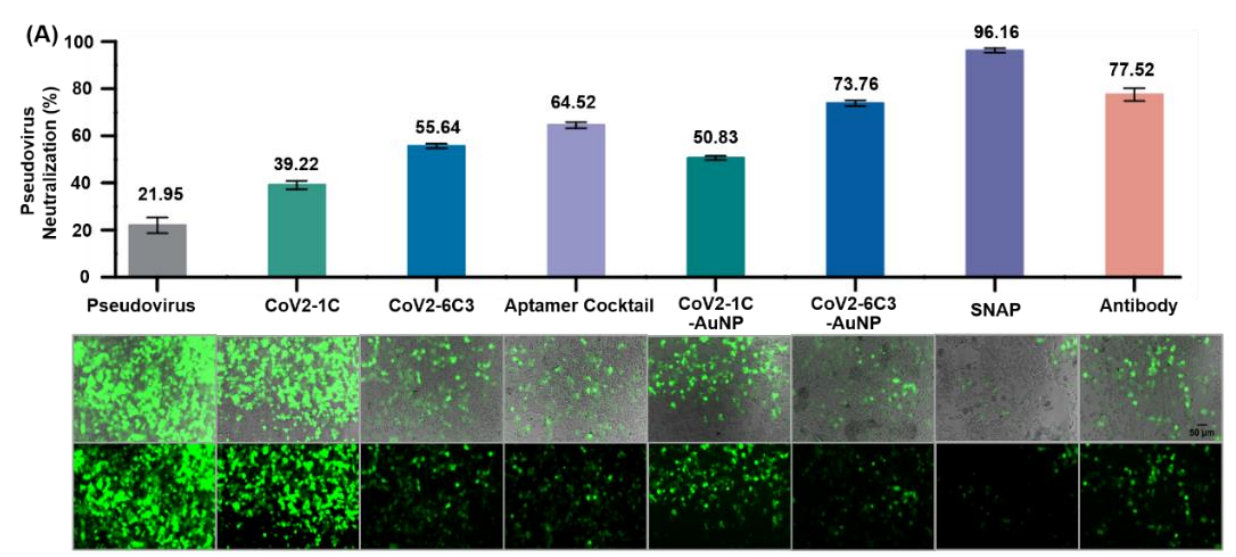

(B)

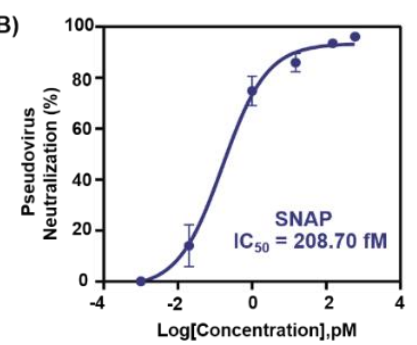

(C)

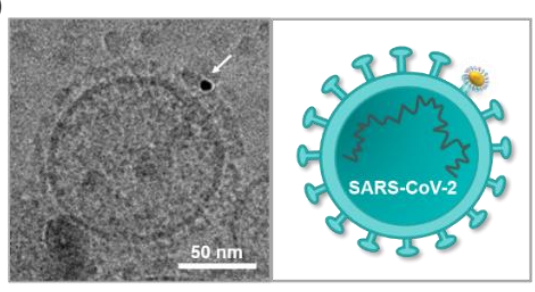

Figure 3. (A) Inhibition of SARS-CoV-2 pseudovirus infection of ACE2-expressing $293 \mathrm{~T}$ cells by $340 \mathrm{nM}$ aptamers and $1 \mathrm{nM}$ SNAP. (B) The neutralization of pseudotyped SARS-CoV-2 virus by SNAP assessed through $\mathrm{IC}_{50}$. (C) The cryo-electron microscopy image (left) and schematic diagram (right) of interaction between SNAP conjugate and SARS-CoV-2 pseudovirus. The concentration of virus is about 100 times the concentration of SNAP. The scale bar is $50 \mathrm{~nm}$.

Recently, two new SARS-CoV-2 variants with N501Y mutation have shown growth rates and effective reproductive numbers in the United Kingdom (501Y. V1) and South Africa (501Y. V2), respectively. Several studies have shown that the two mutated strains have higher transmissibility and severity than that of the original version, are more infectious, and are less susceptible to some existing vaccines or treatment regimens. ${ }^{6,22}$ Therefore, there is an urgent need to develop new strategies 
that can respond to different mutant strains. In addition to the original version of SARS-CoV-2, the SNAP conjugate's binding performance targeting S-RBD and its neutralization ability against pseudotyped virus strain with N501Y mutation were investigated. As shown in Figure 4A, SNAP retains its binding capability targeting S-RBD with N501Y mutation. Therefore, SNAP is expected to be useful for neutralization of the mutated virus strains. As expected, SNAP conjugates block almost 100\% of N501Y mutated pseudotyped viruses from infecting cells (Figure 4B and C).

In addition, the binding affinity and neutralization of SNAP against the pseudotyped virus with D614G mutation ${ }^{4}$ were also evaluated (Figure 4D). SNAP conjugates block more than $96 \%$ of D614G mutated pseudotyped viruses from infecting cells (Figure $4 \mathrm{~F}$ ), with a neutralization $\mathrm{IC}_{50}$ value of 1.24 pM (Figure 4E). However, the aptamer cocktail almost loses its neutralizing ability, probably because the binding modes of the mutated spike-ACE2 complex have changed. Therefore, although the aptamers themselves can bind to the mutated proteins, the binding sites of aptamers on the mutated spike may no longer be the amino acids involved in the binding of mutated spike (D614G) and ACE2. These results suggest that the ideal neutralization capacity of SNAP for different mutated virus strains may be due to the multi-site recognition of cocktailed aptamers and the synergistic-blocking mechanism. Since the inhibition mechanism of the SNAP strategy involves both the conventional neutralization strategy of competing ACE2 binding sites on RBD by the aptamers themselves, and the steric resistance effect of the nano-scaffold, in theory the SNAP strategy still can inhibit virus infection to a certain extent even if the interaction mode of the mutated strain and ACE2 have changed. 

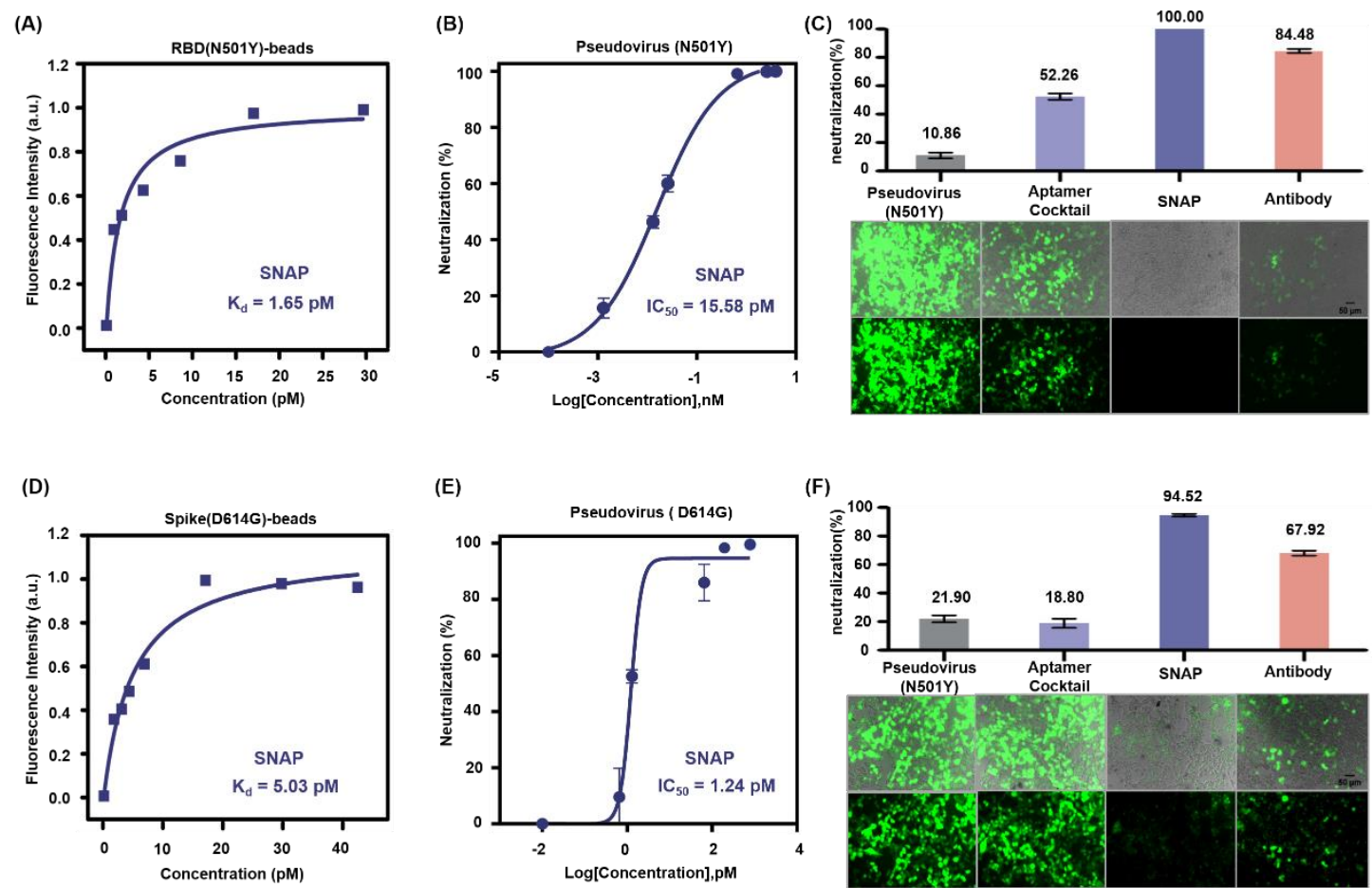

Figure 4. Binding affinity of SNAP against S-RBD with N501Y mutation (A) and Spike with D614G mutation (D). The neutralization of pseudotyped SARS-CoV-2 virus with N501Y mutation (B) and pseudotyped SARS-CoV-2 virus with D614G mutation (E) by SNAP assessed through $\mathrm{IC}_{50}$. Images of SNAP and the antibody neutralization of SARS-CoV-2 pseudovirus with N501Y mutation (C) and D614G mutation (F) infection of ACE2-expressing 293T cells.

To further demonstrate the potential of SNAP for the treatment of COVID-19, the inhibitory activity of SNAP using authentic SARS-CoV-2 with D614G mutation (GenBank: MT835143.1) was assessed. The $\mathrm{IC}_{50}$ value of the authentic SARS-CoV-2 neutralization was determined by quantitative polymerase chain reaction of the SARS-CoV-2 genome in cellular RNA. SNAP conjugates exhibited excellent inhibitory activity against authentic SARS-CoV-2 virus with a half-maximal inhibitory concentration $\left(\mathrm{IC}_{50}\right)$ of 142.8 aM (Figure 5A), 10000- to 10000-fold lower than that of the reported neutralizing antibodies. ${ }^{9,23,3}$ Moreover, the results of immunofluorescent staining by the antibody against SARS-CoV-2 nucleocapsid protein further validated the neutralization effect of SNAP strategy against authentic SARS-CoV-2 virus. As shown in Figure 5B, SNAP protected about $93 \%$ of host cells from SARS-CoV-2 infection. Therefore, the neutralization effects against both the pseudovirus and authentic SARS-CoV-2 virus confirm that SNAP conjugates can inhibit viral entry into the host cell, suggesting that the SNAP strategy has great 
potential for COVID-19 prophylactics and therapeutics.

(A)

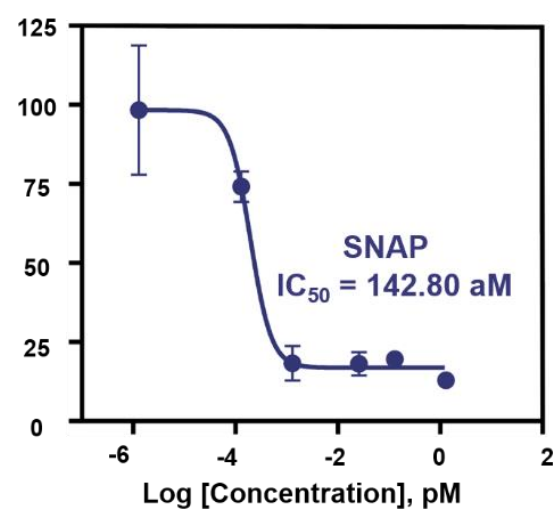

(B)

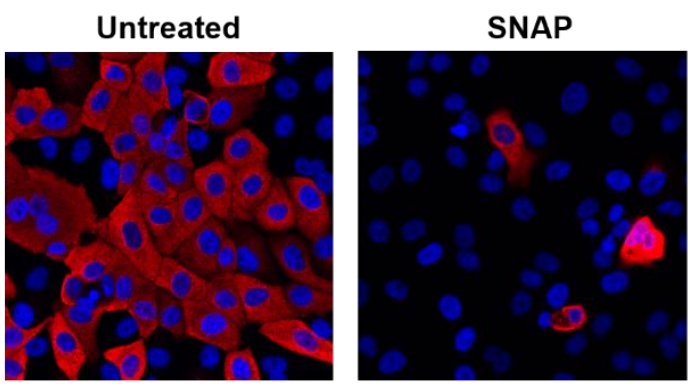

Figure 5. (A) The neutralization of authentic SARS-CoV-2 virus by SNAP assessed through $\mathrm{IC}_{50}$. (B) Images of SNAP neutralization of authentic SARS-CoV-2 infection of Vero E6 cells. The cells were fixed and stained using an anti-SARS-CoV-2 nucleocapsid antibody for the virus and Hoechst dye for the cell nucleus.

In conclusion, we have developed a synergistical-blocking strategy against trimeric-spike to potently inhibit SARS-CoV-2 infection using a spherical neutralizing aptamer with several key advantages. First, homoand hetero-neutralizing aptamers themselves can simultaneously block distinct epitopes on RBD which are involved in the interaction with ACE2. Second, the $5 \mathrm{~nm}$ gold scaffold matches the size of the trimeric-spike to ensure that the neutralizing aptamers enable multi-homologous and multiheterogenous recognition and thus promote simultaneous binding to the three RBDs on the trimeric-spike, but also provides steric hindrance to block the interaction of RBD-ACE2. Based on the synergistical-blocking mechanism, SNAP strategy is vastly capable of neutralizing pseudotyped and authentic SARS-CoV-2, and even the N501Y or D614G mutant strains. Moreover, the U.S. Food and Drug Administration has approved several AuNP-based therapeutic strategies because of the non-toxic and nonimmunogenic nature of AuNPs. ${ }^{24}$ Based on the previous complete biosafety studies on AuNP, the clinical application of SNAP strategy for SARS-CoV-2 therapy may be accelerated. Therefore, the SNAP strategy holds great potential for SARS-CoV-2 prevention and treatment, as well as for in-depth research and development of new anti-virus infection mechanisms, which could greatly assist in preventing future unknown pandemics. 


\section{References}

1 Yan, R. et al. Structural basis for the recognition of SARS-CoV-2 by full-length human ACE2. Science 367, 1444-1448 (2020).

2 Walls, A. C. et al. Structure, Function, and Antigenicity of the SARS-CoV-2 Spike Glycoprotein. Cell 181, 281-292 (2020).

$3 \mathrm{Ju}$, B. et al. Human neutralizing antibodies elicited by SARS-CoV-2 infection. Nature 584, 115-119 (2020).

4 Korber, B. et al. Tracking Changes in SARS-CoV-2 Spike: Evidence that D614G Increases Infectivity of the COVID-19 Virus. Cell 182, 812-827 (2020).

5 Mallapaty, S. What's the risk of dying from a fast-spreading COVID-19 variant? Nature 590, 191-192, doi:10.1038/d41586-021-00299-2 (2021).

6 Wang, Z. et al. mRNA vaccine-elicited antibodies to SARS-CoV-2 and circulating variants. Nature, doi:10.1038/s41586-021-03324-6 (2021).

7 Zost, S. J. et al. Potently neutralizing and protective human antibodies against SARS-CoV2. Nature 584, 443-449 (2020).

8 Cao, Y. et al. Potent Neutralizing Antibodies against SARS-CoV-2 Identified by HighThroughput Single-Cell Sequencing of Convalescent Patients' B Cells. Cell 182, 73-84 (2020).

9 Liu, L. et al. Potent neutralizing antibodies against multiple epitopes on SARS-CoV-2 spike. Nature 584, 450-456 (2020).

10 Song, Y. et al. Discovery of Aptamers Targeting the Receptor-Binding Domain of the SARS-CoV-2 Spike Glycoprotein. Anal. Chem. 92, 9895-9900 (2020).

11 Sun, M. et al. Aptamer Blocking Strategy Inhibits SARS-CoV-2 Virus Infection. Angew.Chem. Int. Ed., doi:https://doi.org/10.1002/anie.202100225.

12 You, M. et al. DNA probes for monitoring dynamic and transient molecular encounters on live cell membranes. Nat. Nanotechnol. 12, 453-459 (2017).

13 Zhao, S. et al. A DNA origami-based aptamer nanoarray for potent and reversible anticoagulation in hemodialysis. Nat. Commun. 12, 358 (2021).

14 Li, S. et al. A DNA nanorobot functions as a cancer therapeutic in response to a molecular trigger in vivo. Nat. Biotechnol. 36, 258-264 (2018).

15 Cutler, J. I., Auyeung, E. \& Mirkin, C. A. Spherical Nucleic Acids. J. Am. Chem. Soc. 134, 1376-1391 (2012).

16 Auyeung, E. et al. Synthetically programmable nanoparticle superlattices using a hollow three-dimensional spacer approach. Nat. Nanotechnol. 7, 24-28 (2012).

17 Benton, D. J. et al. Receptor binding and priming of the spike protein of SARS-CoV-2 for membrane fusion. Nature 588, 327-330 (2020).

18 Mirkin, C. A., Laramy, C. \& Skakuj, K. The Power of Spheres. Nature 576, S3-S6 (2019).

19 Liu, B. \& Liu, J. Freezing Directed Construction of Bio/Nano Interfaces: Reagentless Conjugation, Denser Spherical Nucleic Acids, and Better Nanoflares. J. Am. Chem. Soc. 139, 9471-9474 (2017).

20 Chou, L. Y. T., Zagorovsky, K. \& Chan, W. C. W. DNA assembly of nanoparticle superstructures for controlled biological delivery and elimination. Nat. Nanotechnol. 9, 148-155 (2014). 

Leung, K., Shum, M. H., Leung, G. M., Lam, T. T. \& Wu, J. T. Early transmissibility assessment of the N501Y mutant strains of SARS-CoV-2 in the United Kingdom, October to November 2020. Euro Surveill. 26, doi:10.2807/1560-7917.Es.2020.26.1.2002106 (2021).

23 Bracken, C. J. et al. Bi-paratopic and multivalent VH domains block ACE2 binding and neutralize SARS-CoV-2. Nat. Chem. Biol. 17, 113-121 (2021).

24 Singh, P. et al. Gold Nanoparticles in Diagnostics and Therapeutics for Human Cancer. Int. J. Mol. Sci. 19, 1979 (2018).

\section{Methods}

Cross-competition experiments. CoV2-1C, CoV2-4C and CoV2-6C 3 were also confirmed to bind to different epitopes of SARS-CoV-2 spike RBD by flow cytometry. First, CoV2-1C labeled with FAM was regarded as the target aptamer, adding equi amounts of CoV2-4C or CoV2-6C3 which had no fluorescence label. Similarly, CoV2-4C and CoV2-6C3 labeled with FAM were successively chosen as target aptamer, and the effect of the addition of the other two aptamer sequences without fluorescent label on the ability of the selected target sequence to bind to RBD was observed. The binding competition ability of CoV2-1C, CoV24C and CoV2-6C3 against SARS-CoV-2 spike RBD was assessed by the fluorescence intensity of fluorescent-labeled target aptamer, when the other two aptamers were added. The percentage of the cross-competition was calculated by the equation (the fluorescence signal of the fluorescent aptamer with the other two non- labeled aptamers - background signal) / the fluorescence signal of the fluorescent aptamer without the other two non-labeled aptamers - background signal).

RBD neutralization. In the SARS-CoV-2 spike $\mathrm{RBD}$ protein neutralization assay, $\mathrm{CoV} 2-1 \mathrm{C}, \mathrm{CoV} 2-4 \mathrm{C}$ and $\mathrm{CoV} 2-6 \mathrm{C} 3$ or random sequence was incubated with $10 \mathrm{ng}$ mouse Fc tagged SARS-CoV-2 spike $\mathrm{RBD}$ at $25^{\circ} \mathrm{C}$ for $30 \mathrm{~min}$. Then, the mixture was added to ACE2-Ni-beads and incubated for another 1 hour. The beads were washed twice and resuspended in $200 \mu \mathrm{L}$ binding buffer with biotin-goat anti-mouse IgG-Fc secondary antibody. After incubation for another $30 \mathrm{~min}$, the beads were 
washed twice and resuspended in $200 \mu \mathrm{L}$ binding buffer with SAPE (Streptavidin, R-Phycoerythrin Conjugate from Invitrogen ${ }^{\mathrm{TM}}$ ).To adjust the background signal, the target beads incubated only with SAPE were also included. After the final incubation and washing, the recovered beads were ready for flow cytometry analysis. The percentage of RBD neutralization in the presence of CoV2-1C, CoV2-4C and CoV2-6C3 was compared to RBD maximal binding, calculating by the equation (1- (the fluorescence signal of the sample with the aptamer incubation background signal) / (the fluorescence signal of the sample without the aptamer incubation - background signal) $* 100 \%$ ). Assays were repeated as $\mathrm{n}=3$ independent replicates.

Pseudovirus neutralization analysis. To determine the neutralizing ability of free aptamers $\mathrm{CoV}-1 \mathrm{C}, \mathrm{CoV}-6 \mathrm{C} 3$, aptamer cocktail and their functionalized AuNPs(CoV-1C-AuNPs, 6C3-AuNPs, cocktailed-AuNPs), the pseudovirus neutralization assay was performed as previously described $^{3-4}$. First, DMEM supplemented with $10 \%$ fetal bovine serum and $5 \mu \mathrm{L}$ pseudoviruses per well was pre-incubated with $340 \mathrm{nM}$ aptamer or $1 \mathrm{nM}$ aptamer-functionalized-paticle at $37^{\circ} \mathrm{C}$. One hour later, the mixtures were added to ACE2-expressing $293 \mathrm{~T}$ cells. After six hours of infection, the culture medium was replaced with fresh medium and then incubated at $37^{\circ} \mathrm{C}$ for another $48 \mathrm{~h}$. GFP expression in infected ACE2-transfected $293 \mathrm{~T}$ cells, which were plated at $1.6 \times 10^{4}$ cells/well in 384-well tissue-culture plates, was determined by fluorescence microscopy. The luciferase activity of ACE2-transfected $293 \mathrm{~T}$ cells, which were plated at $1.0 \times 10^{4}$ cells/well in 96-well tissue-culture plates, was measured on a Multimode Plate Reader (SpectraMax iDS). The resulting curves were analyzed by nonlinear regression. The $\mathrm{IC}_{50}$ values were obtained using Prism software version 8.3.0 (GraphPad). These experiments were performed three times.

Authentic viral neutralization assays. All experiments involving live SARS-CoV-2 (GenBank:

MT835143.1, https://www.ncbi.nlm.nih.gov/nuccore/MT835143) followed the approved standard operating procedures of the University of Hong Kong Biosafety 
Level-3 facility. The mixtures of serially diluted aptamers and real virus were incubated at $37^{\circ} \mathrm{C}$. Following incubation for $1 \mathrm{~h}$, the mixtures were added to Vero E6 cells. After another hour of incubation, the supernatant was removed and replaced with DMEM medium with 5\% FBS. After $48 \mathrm{~h}$ incubation at $37^{\circ} \mathrm{C}$, cells were homogenized in $100 \mu \mathrm{L}$ RNAzol solution for the next analysis. The viral mRNA (S gene) and host gene (GAPDH) were detected in a Light Cycle 480 instrument (Roche), and gene expression was measured by quantitative PCR (RT-qPCR) using SYBR green premix Kit (Takara). The relative copy number of viral mRNA level comapred to the host gene mRNA was analyzed by nonlinear regression to calculate the $\mathrm{IC}_{50}$ value using Prism software version 8.3.0 (GraphPad). For immunofluorescence analysis, Vero E6 cells were fixed and incubated in $70 \%$ ethanol at $4{ }^{\circ} \mathrm{C}$ overnight for permeabilization. The experssion of Nucleocapsid protein from SARS-CoV-2 virus was detected by using $\mathrm{N}$ protein primary antibody (Cat: 40143-MM05TA, SinoBiological) for $1 \mathrm{~h}$ at $37^{\circ} \mathrm{C}$. After wished with PBST, slides were incubated with secondary antibody (anti-mouse, Alexa Fluor® 594) for 1 hour at room temperature. Then slides were mounted with mounting buffer containing DAPI. Images were acquired with LSM780 confocal microscope and analyzed with ZEN software.

\section{Data availability}

The authors declare that the data supporting the findings of this study are available within the paper, and Supplementary Information. Extra data are available from the corresponding authors upon request.

\section{Acknowledgements}

We thank the National Natural Science Foundation of China (22022409, 21735004, 21874089), and the Program for Changjiang Scholars and Innovative Research Team in University (IRT13036).

\section{Competing interests}

The authors declare no competing interests. 